\title{
Los servicios profesionales para las personas con discapacidad. Principios, valores y dilemas éticos
}

\author{
Professional services for people with disabilities. \\ Principles, values and ethical dilemmas
}

\begin{abstract}
Resumen
En este artículo analizamos los principios y valores que guían el ejercicio profesional cuando los usuarios tienen una discapacidad, en el contexto de dos campos de conocimiento: la ética profesional y los derechos humanos. Particularmente, nos enfocamos en la labor de los trabajadores sociales. Abordamos también algunos dilemas éticos que estos profesionales pueden enfrentar en su práctica cotidiana, destacando tres de los más señalados en los estudios sobre personas con discapacidad: el conflicto entre los intereses del usuario, el profesional y la institución; la obtención del consentimiento informado, y la tensión entre la autonomía del usuario y los compromisos del profesional. A lo largo del texto, reiteramos la importancia de contextualizar los principios y valores profesionales a partir de las necesidades de las de las personas con discapacidad, las cuales tienden a ser más específicas que las del resto de la población.
\end{abstract}

\section{Palabras clave}

Personas con discapacidad, ética profesional, derechos humanos, dilemas éticos.

\begin{abstract}
In this paper, we analyze the principles and values that guide the professional exercise when the users have a disability, trying to make a linkage between two fields of knowledge: professional ethics and human rights. Particularly, we focus on social workers. We also discuss some ethical dilemmas that these professional may face in their daily practice, showing three of the most explored in the research on people with disabilities: the conflict between the user's interests, the professional's and the institution's; the informed consent, and the tension between the user's autonomy and the professional's commitments. Throughout the text, we emphasize the importance of contextualizing professional principles and values, based on the needs of people with disabilities, which are usually more specific than those of the rest of the population.
\end{abstract}

\section{Keywords}

People with disabilities, professional ethics, human rights, ethical dilemmas.

\section{Judith Pérez-Castro $<$ jcperez@unam.mx>}

Universidad Nacional Autónoma de México (UNAM). México

\section{Pamela González-Osorio <pgonz.osorio@gmail.com>}

Universidad Nacional Autónoma de México (UNAM). México

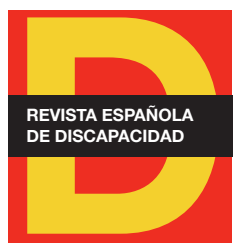

Para citar:

Pérez-Castro, J. y González-Osorio, P. (2018): "Los servicios profesionales para las personas con discapacidad. Principios, valores y dilemas éticos". Revista Española de Discapacidad, 6 (I): 113-137.

Doi: <https://doi.org/10.5569/23405104.06.01.06>

Fecha de recepción: 04-02-2017 Fecha de aceptación: 15-11-2017 


\section{Introducción ${ }^{1}$}

En este artículo buscamos vincular dos campos de conocimiento: los derechos humanos y la ética profesional, para plantear la manera en que los profesionales pueden contribuir a la mejora de la calidad de vida de las personas con discapacidad. La articulación entre estos dos ámbitos no es nueva, de hecho, ésta ha sido trabajada desde varias perspectivas, siendo algunas de ellas: 1) la formación de los nuevos profesionales (Bolívar, 2017; Jorquera, 2014; Vila y Cantero, 2017); 2) el ejercicio profesional (Encinas, 2014; Ife, 2012; Schalock, 2015); 3) la prestación de los servicios de salud y de asistencia social (Cárcar, 2017; Comoretto y Centeno-Cortés; 2016; López et al., 2015); 4) el respeto a los derechos y el logro de la justicia social (Grant y Leigh, 2013; Pearson et al., 2016), y 5) la atención a individuos o grupos en situación de vulnerabilidad (Morris, 2001; García, 2015; López, 2016).

El texto que aquí presentamos se inscribe justamente en esta última línea y tiene como fin analizar los principios y valores que intervienen en la prestación de los servicios profesionales para las personas con discapacidad, así como algunos de los dilemas éticos que se pueden enfrentar cuando el ejercicio está dirigido a estos usuarios.

Cabe mencionar que este trabajo tiene como contexto el debate que se ha venido desarrollando en México desde hace un par de décadas sobre la formación profesional y el papel que ocupan las instituciones de educación superior en este proceso (Díaz-Barriga et al., 2016). En nuestro país, el tema de la ética y los valores profesionales es relativamente reciente. A pesar de la importancia que en su momento tuvieron algunos trabajos pioneros (Maggi, 1997; Muñoz y Rubio, 1993; Ríos, 1997; Spitzer, 1990), hasta finales de los años 90 del siglo pasado todavía no se había logrado consolidar dentro de las líneas prioritarias del campo de la investigación educativa (Yurén et al., 2013: 45).

No obstante, en las primeras dos décadas del 2000, las investigaciones sobre ética profesional se multiplicaron. De acuerdo con el último estado del conocimiento, editado por el Consejo Mexicano de Investigación Educativa, entre el 2002 y el 2012 la producción pasó de 5 a 287 trabajos entre libros de autor, artículos, capítulos de libro, tesis, ponencias y códigos deontológicos (Hirsch y Pérez-Castro, 2013). Las principales temáticas de las investigaciones, hasta ese momento, se centraban en: el estudio de las competencias, rasgos, creencias y actitudes de ética profesional; la enseñanza de la ética profesional en licenciaturas y posgrados específicos; la ética de la investigación científica y el desarrollo tecnológico; los dilemas éticos y las conductas no éticas, y la ética de la profesión docente (Hirsch y Pérez-Castro, 2013).

En estos últimos cinco años, la producción se ha enfocado en la ética de la profesión docente (Hirsch y Navia, 2015; Navia y Hirsch, 2015; Jurado, 2016), la formación en ética profesional de los estudiantes de licenciatura y posgrado (Díaz-Barriga et al., 2016; Félix y Félix, 2016; González, 2017; López-Calva, 2014 y 2015; Vera et al., 2016) y la relación entre ética profesional y responsabilidad social universitaria (Acosta et al., 2016; López et al., 2016; Pérez-Castro, 2016).

1. Este artículo es producto de una investigación que se desarrolla en el Instituto de Investigaciones sobre la Universidad y la Educación (IISUE) de la Universidad Nacional Autónoma de México y que está financiada por el Programa de Apoyo a Proyectos de Investigación e Innovación Tecnológica (PAPIIT), con clave IN300516. 
A pesar de este desarrollo, son pocos los trabajos en México que abordan la actuación ético profesional de los trabajadores sociales (Aguillón, 2011; Carreón, 2015; Flores y Martínez, 2006; Galeana y Tello, 2010; Tello y Ornelas, 2015), y menos aun los que estudian la labor de estos profesionales cuando sus usuarios son personas con discapacidad (Mancinas et al., 2017; Lázaro y Silva, 2014). Se trata de una línea de estudio que, consideramos, se encuentra en plena construcción.

De este modo, para lograr el objetivo propuesto, se ha organizado el artículo en tres apartados: en el primero, se plantean algunos de los elementos del enfoque de los derechos humanos y su convergencia con el modelo social de la discapacidad, haciendo énfasis en el derecho que tiene cada persona a ser reconocida e incluida en los diferentes ámbitos de la vida social. En el segundo, se analizan los principios y algunos valores ético profesionales en el contexto de la atención a personas con discapacidad, enfocándonos particularmente al trabajo social. En el tercer apartado, se discuten algunos de los dilemas éticos que los profesionales pueden enfrentar cuando la competencia o la autonomía de los usuarios se ven comprometidas por una limitación en sus funciones o estructuras corporales. Finalmente, se presentan algunas consideraciones sobre las problemáticas abordadas a lo largo del texto.

\section{La construcción y desarrollo de la noción de discapacidad}

La discapacidad es un concepto construido histórica y socioculturalmente. Se refiere a una experiencia humana específica, cuya explicación ha cambiado de acuerdo con el énfasis depositado en los diferentes factores que en ella intervienen, ya sean las personas, la sociedad, las oportunidades y las relaciones que los individuos pueden establecer o no, al ser etiquetados con esta palabra (Goffman, 1970).

La distinción entre situación y condición de discapacidad, desarrollada por Pantano, hace referencia a la “...vivencia individual, particular y a la manifestación de un fenómeno social caracterizable" (2009: 89). Esta mirada, recuperada y reformulada por otros autores (Ferrante y Ferreira, 2011; Ferreira, 2007; Rodríguez et al., 2017; Roncoli, 2017; Tamayo et al., 2017), implica conocer cómo viven los sujetos la discapacidad y cuáles son los "factores o circunstancias que tienen que ver con las personas en cuanto estén o puedan estar en condición de discapacidad" (Pantano, 2009: 91). La cuestión va más allá de la manera en que se nombre, dado que:

"La condición de discapacidad está asociada a la falta de acceso a bienes y servicios materiales y simbólicos (educación, trabajo, servicios de salud y rehabilitación, prótesis y órtesis, transportes, libre circulación en el espacio público y privado, ocio, entre otros) [...] El motivo de esta exclusión es la presunción de normalidad acerca de los cuerpos y de los modos de ser y estar en el mundo que conduce a la sanción social de aquellos que son ubicados en los márgenes de la norma" (Venturiello, 2017: 167).

La discapacidad también ha sido pensada, de acuerdo con los principios y valores de cada cultura, hacia uno u otro lado de los siguientes binomios: pureza/impureza, milagro/castigo, salud/enfermedad, normalidad/anormalidad, inclusión/exclusión (Brogna, 2009). Más recientemente, se ha buscado entenderla como 
"la compleja interacción entre los rasgos inherentes a una persona (o de su limitación), y la manera en que dichos rasgos se manifiestan en el contexto en el que se desarrollan los individuos (en los hechos discapacitantes de la limitación)"2 (Riddle, 2017: 16).

Estos pares dicotómicos se derivan fundamentalmente de los modelos de discapacidad, desde donde se han propuesto categorías para explicar las representaciones construidas históricamente (Brogna, 2009; Palacios, 2008; Sandoval et al., 2017), que se manifiestan tanto en las prácticas cotidianas como en las formas en que nos relacionados con aquellos que tienen esta condición.

Por una parte, está el modelo individual, que coloca a la discapacidad en la persona (French y Swain, 2008) y que se divide en dos submodelos: el de prescindencia, que establece una relación con lo sagrado para comprender la discapacidad, ya sea como milagro o como castigo (Brogna, 2009), y el médico-rehabilitador, que ha considerado a la discapacidad como "...una condición negativa que debe ser erradicada...para recuperar o alcanzar un status social completamente humano, a través del acercamiento -en la medida de lo posible- a la normalidad biomédica" (Ferrante, 2017: 46). Ambos submodelos contribuyeron a que estas personas fueran aisladas, excluidas, asistidas o compadecidas. Por otra parte, existen diferentes propuestas de análisis y subdivisiones -el modelo social, el de vida independiente, el de derechos humanos, el biopsicosocial, el de calidad de vida, el ecológico, el de la diversidad funcional, etcétera- pero, de acuerdo con Riddle (2017), la mayoría coincide en posicionarse contra la perspectiva que ve a la discapacidad como un problema individual, sustentada solamente en el saber médico.

Así, a partir de la segunda mitad del siglo XX empieza a surgir una nueva forma de entender la discapacidad, la cual ya no se remite a los rasgos o diferencias de las personas, sino más bien a las condiciones que la producen, como la pobreza, la discriminación, la segregación, los prejuicios, el paternalismo y la degradación de la condición humana. Lo que se busca es garantizar el respeto y el reconocimiento de estos individuos, mediante políticas que reviertan y transformen las desigualdades que perduran en las estructuras sociales (Charlton, 1998; Ferrante, 2015; Palacios, 2017).

En sus inicios, la lucha por los derechos de las personas con discapacidad tuvo dos grandes vertientes. En los Estados Unidos, se pugnó porque la discriminación estuviera prohibida en la ley, defendiendo la tradición de oponerse al destino de ciudadano de segunda clase. Mientras, en el Reino Unido, el debate se centró en asegurar la provisión por parte del estado de los servicios públicos básicos necesarios para lograr una vida independiente (Bariffi y Palacios, 2007; Morris, 1991; Roncoli, 2017).

Una década después, la Unión de Personas Físicamente Deficientes contra la Segregación (UPIAS por sus siglas en inglés) emite los 'Principios Fundamentales de la Discapacidad', los cuales constituyeron la base para el modelo social, impulsado por un grupo de académicos británicos, entre ellos Mike Oliver, Vic Finkelstein y Colin Barnes (Ferrante, 2015; Palacios, 2008). Estos principios denunciaban que era la sociedad la que discapacitaba a las personas como resultado de la ideología, las prácticas, las costumbres y la propia organización social. Con el tiempo, esta elaboración teórica fue tomando otras vertientes, congregando disciplinas que abarcaron diferentes dimensiones de las experiencias que las personas tenían en relación con la discapacidad.

2. Traducción propia. 
Esto dio lugar a lo que actualmente se conoce como el campo de estudios sobre la discapacidad o disability studies. Algunos de ellos ven a la discapacidad como un asunto relacionado con las barreras sociales, otros la analizan como una marca cultural o se interesan por la interacción existente entre la limitación y el entorno, mientras que algunos más explican la discapacidad como un ámbito de interés en materia de derechos civiles, cuyo principal sustento son los derechos humanos (Goodley, 2017; Watson et al., 2012).

Los planteamientos del modelo social de discapacidad y el enfoque de los derechos humanos encontraron armonía entre sí, en gran medida por los valores que los sustentan, como la igualdad en dignidad, derechos y libertades, el reconocimiento de la personalidad jurídica y la no discriminación, así como la manera en que ambos analizan las barreras sociales y promueven el desarrollo de cambios que permitan a la persona "contar con los medios que necesite para desarrollar su propio potencial"(Lidón, 2016: 109). En particular, el enfoque de derechos humanos ha buscado generar instrumentos que lleven a la institucionalización de una estrategia política, desde donde se cuestione la forma en que se han ejercido las libertades y los valores, exigiendo accesibilidad plena para las personas con discapacidad lo que, dicho sea de paso, también ha sido una de las demandas más importantes del modelo social (Lidón, 2016; Quinn y Degener, 2002).

Uno de estos instrumentos jurídicos es la Convención sobre los Derechos de las Personas con Discapacidad (CPDP) (Naciones Unidas, 2006), la cual ha sido fundamental para la definición y aplicación de los derechos civiles, económicos, políticos, sociales y culturales de estos sujetos, en diversos aspectos: primero, por la exigencia que hace a los estados parte para garantizar su ejercicio; segundo, porque brinda elementos para el desarrollo de la legislación y las políticas públicas nacionales, y tercero, porque establece mecanismos para el seguimiento de los derechos a nivel nacional e internacional (Naciones Unidas, 2008).

Sin embargo, la promulgación, firma y ratificación de la Convención es sólo un primer paso hacia un horizonte incluyente, ya que la armonización de los marcos internacionales, nacionales, estatales e institucionales ha sido un proceso parsimonioso y, hasta ahora, no se ha logrado establecer una estrategia jurídica que permita a todos los países firmantes garantizar los derechos, impedir que sean violados y destinar los presupuestos requeridos para protegerlos (Lidón, 2016).

Como denuncia Ferrante, se trata de una cuestión política en la que es importante distinguir entre "los derechos 'en papel' (con la CDPD como horizonte a exigir) y el plano de los derechos 'en las prácticas' (examinando las políticas en concreto y utilizando este material como sustrato de caracterización de la situación de discapacidad y denuncia)". (2017: 57).

Transitar los diferentes niveles, desde los internacionales hasta los locales, que enmarcan la experiencia de la discapacidad como condición y situación conlleva dos procesos. Por una parte, es necesario que la persona con una limitación fortalezca o recupere el control sobre las decisiones de su propia vida y, por otra, es preciso simultáneamente reducir las inequidades estructurales e institucionalizadas. Esto contribuiría a visibilizar la tensión entre los derechos reconocidos y las prácticas sociales, dentro de la nebulosa en la que ambos coexisten, así como a denunciar aquellas prácticas en donde los derechos de estas personas están siendo anulados, restringidos o transgredidos (Molina, 2015).

Específicamente en México, algunos de los obstáculos para la implementación de la Convención sobre los Derechos de las Personas con Discapacidad han sido: la ausencia de marcos legislativos que incluyan 
todos los aspectos que este instrumento abarca; la baja tasa de empleo de las personas con discapacidad y el incumplimiento de la cuota laboral del $3 \%$ en los puestos de gobierno; la ausencia de estadísticas e información sobre la discapacidad y la situación en que viven estos sujetos; la denegación del voto a las personas con discapacidad intelectual y psicosocial agudas, así como la falta de alternativas para el ejercicio de este derecho y, por último, la persistencia de las leyes de tutela e interdicción, sin proponer otros caminos para salvaguardar la capacidad jurídica de este sector de la población (Sandoval et al., 2017).

Necesitar ayuda no significa que la autonomía de los individuos esté siendo menoscabada. Éste es un elemento que queremos destacar de lo expuesto hasta ahora, porque introduce la noción de interdependencia, a partir de la cual se cuestiona la visión del ser humano infalible y con independencia plena. Todos los individuos, a lo largo de nuestras vidas, requeriremos algún tipo de apoyo y su demanda se incrementará bajo ciertas condiciones, como la niñez, las enfermedades o la vejez, o en determinadas situaciones, como el maltrato, la vulnerabilidad específica, la pobreza, la discriminación o la exclusión social (CEPAL, 2017; Dhanda, 2008).

Utilizar el enfoque de derechos humanos para analizar la práctica profesional en el ámbito específico de la atención a las personas con discapacidad implica reconocer el vínculo entre estos dos campos, particularmente en lo que atañe a los principios éticos, los roles, las funciones y las actuaciones de los profesionales. Asimismo, permite observar el carácter dilemático de las profesiones cuando se ponen en tensión los estándares sociales del servicio con las necesidades del usuario, o cuando se generan discrepancias entre los principios de la profesión y los derechos humanos. Finalmente, coadyuva a la implementación de los derechos humanos, como perspectiva ética, para guiar el ejercicio profesional y, al mismo tiempo, para reconocer el valor intrínseco de las personas, contribuyendo a su bienestar, especialmente de los colectivos más vulnerables (Munuera y García-Longoria, 2013; Cubillos-Vega, 2017).

\section{La ética profesional en la prestación de servicios a personas con discapacidad}

El interés por regular la actuación profesional se remite al proceso mismo de institucionalización de las profesiones. Los primeros esfuerzos que se hicieron al respecto se empeñaron en definir una serie de rasgos que permitieran distinguir a las profesiones de otras actividades, así como controlar el reclutamiento, la movilidad y el ejercicio de sus miembros. "Hubo un elenco teleológico en esta lista. Las profesiones fueron exitosas gracias a sus atributos: educación prolongada, conocimiento especializado, asociaciones reguladoras, normas y códigos de éticas desarrollados, etc.”3 (Ackroyd, 2016: 16).

Ya en el siglo XX, la discusión giró inicialmente en torno a los factores que intervenían en el mantenimiento de los monopolios profesionales y, posteriormente, el análisis se centró en el ejercicio profesional cotidiano, así como en los discursos profesionales que elaboraban los sujetos en el marco de las instituciones (Abbot, 1988; Johnson y Gore, 2016). De acuerdo con Saks (2016), en las últimas dos décadas los estudios sobre las profesiones se han diversificado aún más, incluyendo cuestiones como la relación entre los distintos

3. Traducción propia. 
ámbitos del sistema ocupacional, los dispositivos de cierre social, el papel de las empresas privadas en la provisión de servicios profesionales y los procesos de profesionalización y desprofesionalización en el marco de la sociedad del conocimiento.

En este artículo, nos enfocamos particularmente en los servicios profesionales que brindan los trabajadores sociales a individuos que se encuentran en situación de dependencia, específicamente aquellos que tienen una discapacidad. Nos interesa analizar los principios y valores ético profesionales que se ponen en juego cuando el ejercicio está dirigido a "personas que requieren apoyos para poder desarrollar las actividades esenciales de su vida diaria, con el fin de alcanzar una mayor autonomía personal y poder disfrutar con plenitud de sus derechos como ciudadanos." (Viscarret et al., 2016: 252).

Por servicios profesionales entendemos aquellas actividades que los miembros de los grupos ocupacionales realizan de manera organizada, en instituciones públicas o privadas, con base en determinados criterios que les permiten establecer el monopolio de su actividad, los límites de su intervención y les otorgan legitimidad (Wilkinson et al., 2016). Asimismo, coincidimos con Macheridis y Paulsson (2017: 4) en que el trabajo profesional se caracteriza por "el uso de la organización profesional como un referente importante, la creencia en los servicios públicos; la creencia en la auto-regulación; el sentido del llamado de la profesión; y la autonomía"4.

Es justamente en el debate entre la autonomía y la responsabilidad, la auto-regulación profesional y la regulación institucional, el ejercicio liberal y el asalariado, que surge la pregunta sobre la ética, es decir, la pregunta sobre el bien, no sólo en términos del servicio que aportan los profesionales, sino también del bienestar individual y social (Hortal, 2002). Uno de los enfoques más importantes desde donde se ha analizado la actuación ético profesional ha sido el principialismo (Israel, 2015; Idareta et al., 2017). Su persistencia y relevancia en el campo se debe, en gran medida, a que éste "puede ser visto como ejemplo de la aplicación de principios éticos generales a contextos particulares"5 (Tapper y Millett, 2014: 62).

El principialismo tiene sus orígenes en los años 70, sus dos fuentes más importantes fueron los 'Principios éticos y pautas para la protección de los seres humanos en la investigación', mejor conocido como el Informe Belmont, y el trabajo de Beauchamp y Childress sobre los 'Principios de la Ética Biomédica' (Beauchamp, 2010; Israel, 2015). Los cuatro principios son: autonomía, beneficencia, justicia y no maleficencia. En el momento en que se plantearon, se pretendía que estos fueran lo suficientemente claros para guiar el trabajo de los diferentes campos profesionales y, al mismo tiempo, lo suficientemente generales para que pudieran ser interpretados desde diferentes tradiciones ético morales (Beauchamp, 2016).

Pero, ¿cómo se expresan estos principios cuando los usuarios tienen una discapacidad?, ¿qué pueden hacer los profesionales para proteger los derechos de estas personas? y ¿cuáles son los principales dilemas que se pueden presentar?

La relación profesional, al igual que el resto de los encuentros que tenemos en la vida cotidiana, es algo que construimos las personas, para lo cual utilizamos la información que aporta cada uno de los participantes, así como el conocimiento previo socialmente construido (Goffman, 1970). La interacción entre un individuo

4. Traducción propia.

5. Traducción propia. 
con discapacidad y otro sin discapacidad no tendría por qué ser distinta, sin embargo, el modelo de normalidad predominante ha hecho que "en nuestras sociedades la discapacidad siga siendo considerada como un disvalor o un 'no valor"” (Etxeberría et al., 2013: 9).

En la relación profesional, lo que está en riesgo no sólo es la provisión del servicio, sino el bienestar del usuario. Por ello, es importante contextualizar los principios profesionales a partir de sus necesidades y capacidades. El principio de autonomía conlleva dos obligaciones: la primera, de tipo negativo, se refiere a la imposibilidad de que las acciones de una persona sean coartadas por otros, mientras que la segunda, de tipo positivo, apunta al fortalecimiento de la elección autónoma de los sujetos, así como el tratamiento cuidadoso de la información (Beauchamp y Childress, 2013). Pero cuando se trata de individuos con discapacidad, esta segunda dimensión tiende a ser vulnerada con el argumento de que no pueden solventar sus necesidades de manera independiente o que carecen de competencia para tomar decisiones. Sin embargo, aquí el trabajo profesional:

"[...] se prolonga en autonomía del beneficiario, de la persona con discapacidad, haciéndola lo menos discapacitada posible a partir de lo cual se va entrando con ella en relación con grado significativo de autonomía por su parte, desde la que ella acoge libremente lo que la acción benefactora de familiares y profesionales le sigue ofreciendo" (Etxeberría, 2005: 54).

El segundo principio, la beneficencia, apela a actuar conforme a los bienes intrínsecos de la profesión y a realizarlos de la mejor manera posible, a través de los conocimientos y los medios técnicos disponibles (Hortal, 1994). No obstante, cuando la competencia del cliente o usuario se ve comprometida, se requiere tener un mayor cuidado de la dignidad de la persona, potenciar sus capacidades, establecer las bases para darle el trato y la información adecuados y garantizar su participación en la consecución del bien intrínseco (Etxeberría et al., 2013).

Por su parte, el principio de justicia nos recuerda que la sociedad es ante todo una empresa cooperativa y que los profesionales deben contribuir a la distribución equitativa y eficiente de los beneficios y obligaciones que se le atribuyen a cada persona (Beauchamp, 2016; Mamo y Fishman, 2013). Asimismo, estos deben comprometerse con el trato justo de las personas con discapacidad y el logro de mejores condiciones de vida, porque sus necesidades y demandas son más específicas que las del resto de las personas. En especial, se debe procurar evitar el maltrato y la negligencia que resultan de la reproducción de prácticas y contextos injustos.

Finalmente, el principio de no maleficencia cuida de que la actuación profesional no genere daño alguno a las personas, ya sea físico, emocional o legalmente. Su contraparte es la beneficencia, no obstante, esta última implica "emprender medidas para ayudar - a prevenir el daño, eliminar el daño, y promover el bienmientras que la no maleficencia requiere solamente la restricción intencional de acciones que causan daño"6 (Beauchamp y Childress, 2013: 152). En la población con discapacidad, la probabilidad de sufrir algún daño, intencional o involuntariamente, se incrementa por la situación de vulnerabilidad en la que se encuentran estos sujetos, pero también por las ideas y prácticas que socialmente se han construido en torno a ellos, así como por la ausencia de marcos legales eficientes para proteger sus derechos (Uribe y González, 2008).

6. Traducción propia. 
Como hemos dicho, el principialismo ha sido un enfoque dominante en los análisis de la actuación profesional, pero la ética profesional no se agota en él. De hecho, cada vez hay más trabajos que abogan por el uso de otras perspectivas, como la ética de las virtudes, la ética de las relaciones y la ética del cuidado (Banks, 2016; Breakey y Sampford, 2017; Cobo 2001). Adicionalmente, los códigos de ética poco a poco han buscado pasar del nivel meramente normativo al procesal y de la definición abstracta de las obligaciones sociales a los compromisos que contraen los sujetos que toman parte del ejercicio profesional (Vanclay et al., 2013).

Específicamente sobre los profesionales del trabajo social, Bermejo (2002) señala que el bien intrínseco que lo sustenta es la construcción de una sociedad en la que cada individuo pueda desarrollarse plenamente, por lo que su principal función debe ser impulsar y fortalecer las capacidades de sus clientes o usuarios, así como trabajar en la eliminación de los factores sociales que los limitan.

Por consiguiente, el ejercicio profesional del trabajador social se ha ampliado más allá de la intervención, para abarcar problemáticas de los ámbitos social y cultural (Pérez, 2007). De este modo, Viscarret et al. (2016), a partir del análisis de lo que los trabajadores sociales 'hacen', han identificado doce ámbitos que dan cuenta de la diversidad de funciones que paulatinamente han asumido estos profesionales, como el trabajo con conductas socialmente desviadas; la atención a las necesidades sociales; la intervención socioeducativa con la familia e infancia/adolescencia; la labor docente o como investigadores; la atención a inmigrantes y/o refugiados; el trabajo en el campo de la salud y la dependencia; la gestión y planificación de los servicios sociales; la atención primaria específica; la inserción e integración social; la intervención directa; la atención a la mujer, y el trabajo con problemáticas diversas en la administración pública.

Particularmente, cuando se trata de individuos que están en situación de dependencia, como las personas con discapacidad, los trabajadores sociales tienen una labor esencial en la identificación y superación de las barreras que los vulneran y excluyen socialmente. Por esta razón, su ejercicio profesional necesita sustentarse en principios y valores que no sólo les ayuden a orientar su trabajo, sino que también les briden protección a ellos y a sus usuarios. Para Congress (2010), los principios y valores centrales que comparten los códigos de ética del trabajo social pueden agruparse en dos grandes rubros: los relacionados con la práctica profesional (confidencialidad, integridad profesional y autodeterminación) y los relacionados con la dignidad humana y la justicia social (respeto por las personas, no discriminación, competencia cultural, derechos humanos y justicia social).

Uno de los rasgos que distinguen al trabajo social es que su ámbito de intervención se sitúa en los espacios de interacción de los sujetos. Para lograr el cambio social y promover el empoderamiento de los individuos, Munuera (2012) plantea los siguientes principios:

1. Comprensión de las necesidades y capacidades de las personas con discapacidad, como compromiso básico de justicia.

2. Desarrollo de la red social a través del fortalecimiento de los individuos y grupos.

3. Respeto a los derechos, la justicia, la tolerancia, la autodeterminación, el desarrollo personal y la seguridad familiar.

4. Compromiso con la ética, los valores y la práctica del trabajo social. 
El objetivo final de la intervención profesional en este tipo de situaciones es "fortalecer la habilidad de las partes en conflicto con miras a lograr un arreglo duradero, aceptado por ambas partes, tomando en consideración las necesidades de cada miembro, distinguiendo las posiciones y señalando los intereses comunes que pueda haber entre ellos"7 (Munuera, 2015: 16).

Otros principios que, desde la perspectiva de Dolgoff et al. (2012), deben considerar los trabajadores sociales son: la protección de la vida humana, el trato justo a las personas, el fortalecimiento de la autodeterminación, la autonomía y la libertad de los usuarios, evitar el daño, el mejoramiento de la calidad de vida, el respeto a la privacidad y la confidencialidad, y hablar siempre con la verdad. El orden de estos principios no está predeterminado, sino que en cada situación los profesionales deben ponderar la prioridad que le dan a cada uno, especialmente en aquellas circunstancias en donde dos o más de ellos pueden generar alternativas en conflicto.

Finalmente, Fernández y Ponce (2014) plantean seis principios para orientar la intervención del trabajador social:

1. Consideración individualizada. Su punto de partida es el respeto y el reconocimiento del valor único que tiene cada persona. Este principio exige del profesional rasgos específicos como: la minuciosidad, la objetividad, el entendimiento y la flexibilidad, así como saber escuchar y observar.

2. La aceptación del usuario. Como su nombre lo indica, significa aceptar a la persona como realmente es, sin que esto merme la objetividad del profesional. Este principio requiere del respeto, la receptividad, el interés, el análisis y comprensión de los esquemas de acción, y la capacidad de discernimiento entre las necesidades del usuario y las del trabajador social.

3. La autodeterminación. Conlleva el respeto y la aceptación de la libertad de cada persona para tomar sus propias decisiones. Es decir, potenciar las capacidades del usuario sin tratar de ocupar su lugar ni generar dependencias. La labor del trabajador social consiste en ayudar al usuario a aclarar su situación, proveerle información adecuada, mostrarle los recursos disponibles y construir un clima propicio para que se exprese con libertad y confianza.

4. No enjuiciamiento. El profesional debe abstenerse de emitir opiniones, valoraciones y consideraciones sesgadas sobre el caso en el que interviene y mucho más sobre las personas involucradas en él. Su función es “...comprender las debilidades y fracasos de los usuarios, pero nunca deberá juzgarlos. El derecho y las autoridades competentes son los encargados de emitir juicios y sentencias" (Fernández y Ponce, 2014: 51).

5. La expresión y combinación de sentimientos. El usuario es ante todo una persona con sentimientos, que se combinan de formas diversas dependiendo de la situación que enfrenta y que, bajo ciertas condiciones, lo pueden llevar a la inestabilidad emocional. El trabajador social debe ayudar a crear un clima adecuado que le permita expresar sus sentimientos y mantener su equilibrio. Los dos rasgos fundamentales aquí son la sensibilidad y la comprensión.

6. Confidencialidad. Este principio es "un deber del trabajador social con casos y un derecho del cliente mediante el cual se garantiza la intimidad y el secreto profesional de toda la información manifestada durante

7. Traducción propia. 
la intervención" (Fernández y Ponce, 2014: 51). Para ello, el trabajador social debe tener en cuenta los principios profesionales, los límites institucionales de su actuación e, incluso, su propia intuición, sobre todo en aquellas circunstancias en donde esté en riesgo la vida o integridad de los usuarios o clientes.

\section{Dilemas éticos en la atención a personas con discapacidad}

Los dilemas éticos son ineludibles en el ejercicio profesional y en la vida misma. Continuamente, las personas tenemos que tomar decisiones en las que nos vemos obligados a elegir entre dos posibles cursos de acción, que se contraponen entre sí y que conllevan una serie de consecuencias morales, económicas, sociales, políticas e, incluso, jurídicas.

Según Resnik (1998), los dilemas son situaciones en las que las normas y valores entran en conflicto. Su solución suele generar incomodidad y molestia, porque para ambos lados del problema pueden existir suficientes argumentos a favor o en contra, por lo que las personas suelen tener muchas dudas sobre lo que se 'debe' y lo que se 'puede' hacer.

Por su parte, Reamer (2013: 4) sostiene que "un dilema ético es una situación en la cual los deberes y las obligaciones profesionales, enraizados en valores centrales, chocan. Aquí es cuando los trabajadores sociales deben decidir qué valores - según se expresan en diferentes deberes y obligaciones- tienen preminencia"8.

De manera más específica, Banks y Williams hacen una distinción entre problema ético y dilema ético. El primero es "una historia acerca de una situación compleja, en donde se tuvo que asumir una decisión, pero en la que no había ningún dilema para la persona que tomó la decisión - es decir, se tenía claridad sobre el curso de acción a seguir -"9 (2005: 1011).

Por el contrario, un dilema ético es:

"[...] una historia acerca de la toma de decisiones, que implica la difícil elección entre dos alternativas igualmente inoportunas y en donde no está claro cuál de ellas es la correcta. Una situación o evento por sí mismo no es un dilema, pero puede construirse como dilema para ciertas personas" (2005: 1011)"”10.

Las situaciones dilemáticas en el trabajo profesional pueden ser diversas, sin embargo, cuando la competencia del usuario o cliente se ve disminuida, esta diversidad puede ser mayor, dependiendo de la limitación que tenga la persona, así como de la relación que se haya logrado construir entre ella, el profesional y su familia. En cualquier caso, como advierte Reamer (2014), los dilemas exigen un análisis profundo de la situación, en donde, entre otras cosas, se ponen en juego los valores, deberes y obligaciones profesionales.

8. Traducción propia. 
El trabajo social es particularmente una profesión en donde se enfrentan numerosos dilemas éticos "dado que, no sólo se trata con personas, sino que también interviene en situaciones y contextos -a veces muy delicados - en los que se ven inmersas esas personas" (Ballestero et al., 2014: 228).

Justamente con el fin de analizar los principales problemas éticos que se presentan en el ejercicio profesional, Ballestero et al. (2013) realizan una investigación con una muestra de trabajadores sociales en España. Los resultados les permitieron identificar siete tipos de dilemas concernientes a:

1. La protección y el mantenimiento de la confidencialidad.

2. La información que los profesionales brindan a los usuarios.

3. Los límites de la autonomía de los usuarios.

4. Los límites de la confidencialidad sobre los usuarios.

5. Los vínculos que establecen el profesional, los usuarios y los colegas.

6. El respeto a las opiniones de los usuarios y el acceso de la historia social.

7. La relación entre el trabajador social y los usuarios.

Otros dilemas en el trabajo social están relacionados con (Ballestero et al., 2012):

- La información que se le proporciona a terceras personas.

- La duración de la intervención ya sea porque se tiene que cerrar el caso, a pesar de las implicaciones que esto tiene para los individuos involucrados, o por el poco tiempo que en ocasiones se puede dedicar a la atención de los usuarios.

- Los problemas que se generan por las malas prácticas de otro trabajador social u otros profesionales.

- El manejo de situaciones en donde el trabajador social descubre que los usuarios han proporcionado información parcial o inexacta, con el fin de continuar recibiendo el servicio.

- Los conflictos relacionados con los criterios para la distribución de apoyos y recursos, especialmente con usuarios en situación de vulnerabilidad

- Las dificultades originadas por el contenido y redacción de los informes sobre los usuarios, así como por la titularidad y el acceso a los mismos.

- La obtención del consentimiento informado.

- Las circunstancias en donde los usuarios ofrecen alguna retribución material o económica al trabajador social para obtener algún servicio o prestación.

Por su parte, Dolgoff et al. (2012) sostienen que, de manera general, los dilemas éticos pueden diferenciarse entre aquellos que se originan por valores en conflicto (competing values), por ejemplo, cuando el profesional se ve obligado a elegir entre la justicia y la equidad o entre la confidencialidad y la protección de la vida del usuario, y aquellos que se originan por el conflicto entre lealtades (competing loyalties), en donde el trabajador social es presionado por diferentes personas o grupos con intereses y demandas contradictorias entre sí. 
Con base en lo anterior, los autores plantean los siguientes dilemas ético profesionales (Dolgoff et al., 2012):

1. El conflicto entre la autonomía del cliente y la expertise profesional.

2. Diferencias entre los valores del usuario y el profesional.

3. Diferencias entre los intereses del cliente, el profesional y la institución en donde se brinda el servicio.

4. Decir la verdad, sin manipular, presionar o hacer sentir mal al cliente y, al mismo tiempo, evitar que él malinterprete o tenga una falsa idea de su situación.

5. Problemas derivados de un mal diagnóstico.

6. Mantener la confidencialidad sobre la situación del usuario frente a las demandas de información de terceros involucrados.

7. Lograr el consentimiento informado.

8. Problemas en el aprovechamiento y distribución de los recursos cuando estos son limitados.

9. Problemas en la intervención y defensa de los recursos, servicios y derechos del usuario.

10. Conflictos por las malas prácticas de los colegas u otros profesionales.

11. Diferencias culturales o religiosas entre el cliente y el profesional.

12. Problemas entre las demandas o necesidades del usuario y el cumplimiento de las políticas y regulaciones institucionales.

De los diferentes dilemas, nos interesa destacar tres: el conflicto entre los intereses del usuario, el profesional y la institución, la obtención del consentimiento informado, y la tensión entre la autonomía del usuario y la del profesional. Estos han sido señalados entre los más relevantes tanto en las investigaciones sobre personas con discapacidad (Lépiz 2000; Leturia et al., 2014; Muñoz y Lucero, 2014; Román, 2010; Ramos, 2013; Rodríguez-Arias y Rodríguez-Arias, 2005; Rueda y Miranda, 2002), como en otros colectivos en situación de vulnerabilidad (Ballestero et al., 2012; Beauchamp y Childress, 2013; Busso y León-Sanz, 2016; Congress, 2010; Israel, 2015; Liamputtong, 2007; Parrot, 2010).

Con respecto al primero, cabría decir que cuando el servicio profesional se brinda a través de alguna institución, ya sea un hospital, una escuela o una oficina de asistencia social, los dilemas se producen porque el profesional tiene que lidiar entre el logro del bienestar para el usuario, las exigencias institucionales y sus propios principios y valores. A continuación, presentamos una problemática que se suscitó en un plantel de educación media superior de la Ciudad de México.

En el ciclo escolar 2015-2016, la escuela recibió a un grupo de doce jóvenes con discapacidad auditiva. Como la institución no tenía recursos para atender las necesidades educativas de estos alumnos, los padres acordaron contratar por su cuenta a dos intérpretes de lengua de señas, quienes estarían apoyando a los profesores en las clases. La inclusión de estos chicos significó todo un reto para los maestros y los intérpretes, ya que era la primera vez que trabajaban en una empresa de esta naturaleza, así como para la escuela, que estaba obligada a brindar el servicio. Al final del primer año, sólo siete de los doce jóvenes continuaban estudiando (De la Cruz, 2017). 
El dilema aquí gira en torno a la postura que debe asumir la administración escolar, es decir, si tendría que hablar con los estudiantes y sus padres para que retomen sus estudios, toda vez que en México la educación media es obligatoria desde el 2011, o bien si tendría que dejar pasar la situación pues, en última instancia, fueron los papás quienes asumieron los costos adicionales de la educación de sus hijos. Además, en la descripción que se hace del caso, los maestros y los intérpretes de lengua de señas dijeron desconocer los motivos del abandono escolar, es decir, si ésta había sido una decisión de los estudiantes o de sus progenitores.

La pregunta de fondo es: ¿quién es el usuario: la persona con discapacidad, la institución educativa o los padres? Para Dolgoff et al., (2012), la respuesta no se encuentra en el modelo profesional tradicional.

"Un modelo actualizado puede incluir al solicitante, al cliente, el objetivo, el beneficiario, el profesional, la agencia, la comunidad, y otros [...] A veces, la misma persona es el solicitante, así como el cliente, el objetivo y el beneficiario. En otros momentos o situaciones, diferentes personas ocupan cada una de estas posiciones. El solicitante puede no ser el cliente (por ejemplo, cuando una madre solicita servicios para su hijo) y el cliente no tiene que ser el objetivo (por ejemplo, el objetivo puede ser un empleador o el director de la escuela), ni el cliente es necesariamente el beneficiario de la intervención"11 (Dolgoff et al., 2012: 93).

Otro ejemplo es el que propone Parrot (2010). En él, se narra la situación de una madre de familia que se hace cargo de su hija, quien tiene discapacidad física e intelectual. Esta última asiste a un centro de cuidado, de las denominadas instituciones de relevo que brindan atención integral por períodos determinados y que, como su nombre lo indica, fueron diseñados para relevar temporalmente a los cuidadores familiares y mantener el bienestar, tanto de estos como de las personas con discapacidad. Recientemente, la madre ha pedido un período extra, debido a que su hija sufrió un accidente que le dejó diversas secuelas. El trabajador social que lleva el caso habla con el supervisor, quien considera que las posibilidades de aprobar la petición son bajas, porque el centro de cuidado ha decidido limitar las estancias por persona y también porque otras solicitudes presentadas anteriormente no han sido autorizadas.

El dilema que se presenta en este caso es resultado de la tensión entre de la demanda de la madre, quien solicita el servicio; la necesidad de la persona con discapacidad, que es la beneficiaria directa del centro de cuidado, y la postura de la institución, que ha acotado el número de estancias para optimizar los recursos y permitir que otros puedan beneficiarse.

Para Parrot (2010), hay dos caminos posibles para abordar este dilema. El primero es seguir las normas, lo que desde un enfoque burocrático procedimental significa que el trabajador social acepte la respuesta del supervisor y le notifique a la solicitante del servicio que su petición ha sido denegada. El segundo es asumir una perspectiva flexible reflexiva, que conlleva pensar críticamente sobre los factores que intervienen en el dilema. Esto permite que el profesional vea otros cursos de acción posibles, en los que se puedan conciliar las demandas de las partes involucradas. En este ejemplo el trabajador social podría apelar ante el supervisor la particularidad del caso, o bien ver si existe la posibilidad de asignarle a la persona con discapacidad algún período de estancia que otro usuario no esté ocupando.

11. Traducción propia. 
De cualquier modo, el autor advierte de que el profesional debe tener el cuidado de considerar los objetivos y valores de la institución para la que trabaja y, al mismo tiempo, las condiciones de cada situación, en la que convergen expectativas, valores y deberes del usuario, del profesional y, en este caso, de una tercera persona que es el solicitante.

Otro dilema que se puede presentar cuando se trabaja con personas con discapacidad es la obtención del consentimiento informado. Éste es un procedimiento que durante mucho tiempo se ha utilizado en la práctica médica y que se ha extendido hacia otras disciplinas y profesiones que trabajan con seres humanos. De acuerdo con Grady (2015: 855), el consentimiento informado "es la autorización de una actividad basada en el entendimiento de lo que dicha actividad implica y en la ausencia de control por otros"12. El contenido del documento suele estar sujeto a las leyes y la normatividad de cada país, mientras que los valores en los que éste se sustenta "están profundamente arraigados en la cultura - específicamente, el valor del respeto por la autonomía de la persona y su derecho a establecer sus propias metas y a hacer las elecciones pertinentes para alcanzar dichas metas -"13 (Grady, 2015: 855).

El consentimiento informado implica que los individuos conozcan con claridad la ruta que seguirá la intervención profesional, los beneficios y riesgos que se pueden generar y, como su nombre lo indica, que ellos consientan libremente participar en este proceso. No obstante, en la población con discapacidad esto no siempre es posible, especialmente en aquellas personas con limitaciones severas, lo que se convierte en una fuente de tensión para los profesionales.

Para analizar este tipo de dilemas, Lépiz (2000) describe el caso de una persona con discapacidad psicosocial a quien se le tiene que administrar un tratamiento farmacológico controlado para contrarrestar una crisis que se le ha presentado. No obstante, debido a su condición, el usuario no está en capacidad de otorgar el consentimiento informado. Esto le plantea al equipo médico la disyuntiva de aplicarle el tratamiento, o bien esperar hasta obtener la autorización, ya sea de su parte, de sus padres o de sus tutores legales.

El consentimiento informado es una norma derivada del principio de autonomía, es decir, se requiere que la persona que lo asume tenga conciencia y voluntad suficientes para tomar decisiones (Ballestero et al., 2012; Beauchamp y Childress, 2013). Sin embargo, la discapacidad no inhabilita automáticamente a la persona, ni siquiera cuando están comprometidas las dimensiones cognitivas y psicosocial. Lo primordial es evitar o disminuir la probabilidad de provocar algún daño en el usuario, lo que atañe al principio de no maleficencia, a partir de una valoración cuidadosa de su capacidad que, de ser posible, debe hacerse desde una perspectiva multidisciplinaria (Ramos, 2013).

Conjuntar estos dos principios, autonomía y no maleficencia, no siempre es sencillo, incluso en individuos sin discapacidad. Para Beauchamp y Childress (2013), esta dificultad radica en que, por una parte, la autonomía sólo tiene una obligación prima facie, por lo que puede quedar anulada frente a otras cuestiones. Por otra parte, la no maleficencia comprende diferentes nociones que, aunque están interconectadas, no necesariamente mantienen una relación causal entre sí.

12. Traducción propia.

13. Traducción propia. 
Por ejemplo, el vínculo entre agravio y daño. El primero implica la violación de los derechos de una persona y aunque un agravio generalmente provoca daños, el daño no necesariamente conlleva la realización de un agravio, ya que éste puede ser producto de un accidente, un desastre natural y hasta por mala suerte (Beauchamp y Childress, 2013).

En cuanto al segundo, los autores señalan que un daño siempre produce algún tipo de pérdida, fracaso o disminución de los intereses del individuo, empero, no todo daño es injustificable o erróneo, como cuando se necesita hacer una amputación (Beauchamp y Childress, 2013). Indudablemente, aquí, el sujeto tendrá una pérdida, pero, en la mayoría de los casos, este procedimiento es necesario para resguardar la vida.

Otro ejemplo, en donde la obtención del consentimiento informado resulta problemática, es la esterilización de las personas con discapacidad intelectual o psicosocial. La justificación que los familiares y tutores generalmente dan para realizar este procedimiento es que se intenta evitar la posibilidad de embarazo por abuso sexual (Muñoz y Lucero, 2014). Nuevamente, para esto se requiere el análisis cuidadoso de la situación por parte de diferentes especialistas, a fin de preservar el bienestar del usuario y proporcionarle todos los elementos posibles para que tome una decisión informada. Cuando esto no es posible, el consentimiento informado tendrá que ser otorgado por los padres, tutores o representantes legales. Sin embargo, Muñoz y Lucero (2014: 67) se preguntan: “¿corresponde esterilizar a un adulto con discapacidad intelectual sin su consentimiento?, ¿la esterilización protege a las personas con discapacidad o a sus familias? y ¿quién y cómo evalúa la capacidad para asumir la paternidad o maternidad?".

Sin duda la resolución de este dilema no es sencilla pero, de acuerdo con Román (2010), cuando el usuario es una persona con discapacidad, el consentimiento informado, además de atenerse a los principios de la ética profesional, debe sustentarse en la ética de la justicia y de la solidaridad a partir de tres niveles: el macro, que incluye el análisis de las políticas sociosanitarias y las leyes sobre los servicios sociales; el meso, que abarca la normatividad y las políticas institucionales, y el micro, que es en donde se construye la relación entre el profesional, la persona con discapacidad y su familia o tutores.

El tercer y último dilema que queremos abordar es el que deriva de la tensión entre la autonomía del usuario y el compromiso del profesional con sus propios principios y valores. Como hemos dicho anteriormente, la autonomía tiene dos sentidos: el fortalecimiento de la autodeterminación y la prohibición de que las acciones de un individuo sean restringidas o controladas por otros.

En las personas con discapacidad, una de las situaciones en donde más polémico resulta este principio es en lo referente a la sexualidad (Perlin, 2008), particularmente en aquellas que tienen discapacidad de origen congénito. Al respecto, Hernández (2017) expone la problemática ocurrida en un Centro de Atención Múltiple (CAM) en México, en donde se impartía un taller sobre orientación sexual a adolescentes con discapacidad intelectual. Los padres de familia se oponían a la realización de esta actividad, lo que puso a la trabajadora social de la institución ante el conflicto de preservar la autonomía y el bienestar de estos jóvenes y, al mismo tiempo, enfrentar la presión de los padres, quienes no consideraban apropiado tratar este tipo de temas con sus hijos.

Otro escenario, pero sobre este mismo tema, es el que se presenta en personas con discapacidad adquirida, por ejemplo, cuando se sufre la amputación de un miembro. En muchos casos, el impacto del procedi- 
miento es muy fuerte y a los individuos les toma mucho tiempo aceptarlo. "Algunos de ellos pondrán todo de sí para asumir su nueva condición mientras que otros, estando en rehabilitación, se niegan a mejorar, cursando con depresión y deseando morir" (Rueda y Miranda, 2002: 131). Cuando el usuario se niega a recibir ayuda, el profesional se ve en la disyuntiva de respetar su decisión o de insistir en la rehabilitación.

Como ya decíamos, hay situaciones que pueden anular la primacía del principio de autonomía. Úriz et al. (2013) señalan tres particularmente: cuando se pone en riesgo la vida o la integridad del usuario, cuando se producen daños a terceros o cuando se daña al profesional. Pero, además, la autonomía requiere intencionalidad, entendimiento y estar libre de restricciones, ya sean externas o internas. En estas últimas se incluyen aquellos factores que limitan la autodeterminación, como la discapacidad (Beauchamp y Childress, 2013). Estas tres condiciones siempre están en función de grados, porque no hay una persona que pueda decir que es totalmente libre, que tiene completa claridad de sus intenciones y que posee pleno entendimiento de todas las cosas. De este modo, la autonomía no puede ser vista en términos dicotómicos, como algo que se tiene o no se tiene, sino que debe ser valorada en cada caso.

No se puede descalificar la competencia de la persona por tener una discapacidad, aunque sea de tipo intelectual. Por el contrario, ésta necesita ser evaluada desde diferentes enfoques profesionales y a lo largo del tiempo, ya que puede fortalecerse o deteriorarse (Muñoz y Lucero, 2014). En cualquier caso, como señalan Fernández y Ponce (2014), el profesional tiene que hacer un análisis individualizado de cada situación, aceptar a la persona como es, respetar su autodeterminación, construir un contexto propicio para que exprese sus sentimientos y mantenga su equilibrio emocional, preservar su derecho a la confidencialidad y, sobre todo, no expresar opiniones o juicios sin fundamento que en nada contribuyen al bienestar del usuario.

Finalmente, los profesionales deben tener presente que, si bien no siempre hay una resolución definitiva para los dilemas, esto no los exime de la toma de decisiones (Banks y Williams, 2005). Además, en el caso particular de las personas con discapacidad, el ejercicio de los principios y valores que se conjugan en cada situación dilemática debe hacerse respetando su autonomía y sin atentar contra las exigencias generales de la justicia (Etxeberría et al., 2013).

\section{Consideraciones finales}

La ética profesional es la capacidad de pensar críticamente sobre el saber profesional, pero también sobre los principios y valores que guían la labor de los profesionales (Hortal, 2002). Su finalidad es brindar referentes que permitan reflexionar sobre el trabajo cotidiano, es decir, sobre lo que hay que hacer, quiénes, para qué y para quiénes (Etxeberría, 2016).

En este artículo hemos abordado los principios y valores que los trabajadores sociales deben considerar cuando su ejercicio está dirigido a personas con discapacidad, así como algunos de los dilemas éticos que se pueden presentar. Esto no significa que se tengan que construir pautas distintas para estos sujetos, por el contrario, siguiendo a Congress (2010), podemos decir que los principios y valores no sólo son comparti- 
dos por diversos campos profesionales, sino que además son válidos independientemente de la capacidad de los usuarios. Con todo, es importante contextualizarlos a partir de las necesidades de cada individuo, pues en el caso de las personas con discapacidad sus demandas suelen ser más específicas y tienden a estar expuestas a una mayor vulnerabilidad, la cual ha sido construida socialmente, institucionalizada y persistente hasta la fecha en varias de sus manifestaciones. Asimismo, es necesario establecer un diálogo entre el saber profesional, las estrategias de la familia para apoyarlo y, sobre todo, el conocimiento experto que la persona tiene sobre su discapacidad lo cual, como señalan Etxeberría et al. (2013) y Munuera (2012), constituye un compromiso fundamental de justicia que los profesionales deben hacer suyo.

Pero en el ejercicio profesional también se enfrentan dilemas que ponen en juego los principios y valores de los sujetos que toman parte y que, en ocasiones, acotan sus posibilidades de acción. De hecho, en las últimas décadas, ha habido un mayor interés por comprender este tipo de situaciones, las cuales parecen haberse diversificado tanto por factores propios de los procesos de profesionalización y configuración de los mercados laborales, como por la intensificación de los cambios sociales (Congress y McAuliffe, 2006; Reamer, 2014; Saks, 2016)

Los dilemas son construcciones que los individuos hacen con base en los factores y condiciones que en ellos intervienen, es decir, no es que haya situaciones que sean de naturaleza eminentemente dilemática y otras que no lo sean. Igualmente, aunque todas las profesiones tienen sus propios dilemas, en el trabajo social estos toman un matiz particular porque se conjugan sujetos, circunstancias específicas y contextos muy diversos (Ballestero et al., 2014). Los dilemas ponen a prueba la perspectiva flexible reflexiva que el profesional puede asumir cuando analiza los posibles cursos de acción que puede llevar a cabo.

Aquí hemos abordado tres que han sido destacados en la literatura sobre la población con discapacidad: el conflicto entre los intereses del usuario, el profesional y la institución, la obtención del consentimiento informado y la tensión entre la autonomía del usuario y la del profesional. Los dos últimos atañen a los principios profesionales de no maleficencia y autonomía, mientras que el primero tiene que ver con los diferentes sujetos o agentes que participan en la relación profesional.

El tema de la autonomía resulta particularmente problemático en circunstancias en donde concurren individuos con discapacidad, porque se tiende a poner más atención en sus limitaciones que en sus capacidades, en especial cuando las dimensiones cognitivas y psicosociales son afectadas. En ocasiones, los padres o incluso los profesionales pueden disminuir la autodeterminación de las personas, con o sin proponérselo, en lugar de ayudarles a comprender su situación y a tomar sus propias decisiones, como sucede en aquellas situaciones en donde se necesita obtener el consentimiento informado.

En cualquier caso, los profesionales deben tener presente que su principal interés no está en las circunstancias específicas que limitan las capacidades del usuario, sino más bien en el logro y la preservación de su bienestar. Enfrentarse a un dilema ético compromete a los profesionales a tomar una decisión; pueden decidir cumplir con lo normativo y mantener el orden social, o bien pueden ser agentes de cambio y reflexionar sobre cursos de acción diferentes que contribuyan a mejorar su trabajo y a entender las necesidades de las personas, al darle un lugar relevante a sus propias voces. Finalmente, aunque la decisión que se tome ante un dilema puede generar más dudas que certezas, los profesionales siempre deben reflexionar cuidadosamente sobre los principios y valores implicados, así como sobre las consecuencias, reversibles o irreversibles, de los diferentes caminos que éste plantea. 


\section{Referencias bibliográficas}

Abbott, A. (1988): The system of professions. An essay on the division of expert labor. Chigago: The University of Chicago Press.

Ackroyd, S. (2016): "Sociological and organizational theories of professions and professionalism", en: Dent, M. et al. (coords.): The Routledge Companion to the Professions and Professionalism. Abingdon, U. K.: Routledge.

Acosta, A. et al. (2016): "Representaciones sobre la responsabilidad docente en estudiantes de posgrado en educación en México", en Izarra, D. (coord.): Experiencias y perspectivas en ética profesional y responsabilidad social universitaria en Iberoamérica. Táchira, Venezuela: Universidad Pedagógica Experimental Libertador Instituto de Mejoramiento Profesional del Magisterio.

Aguillón, I. (2011): Código de ética, moral y calidad de vida del trabajador social. México: UAEH - Lito-Grapo.

Ballestero, A. et al. (2014): "Cómo afrontar los dilemas éticos en la práctica profesional del trabajo social”, en Seller, E. (ed.): Las prácticas curriculares en el Grado de Trabajo Social. Supervisión y construcción del conocimiento desde la práctica profesional. Madrid: Editorial Universitas.

Ballestero, A. et al. (2013): "Cuestiones éticas y tipologías de comportamiento ético en la intervención profesional del Trabajo Social”. RES. Revista española de sociología, (19): 67-92.

Ballestero, A. et al. (2012): "Dilemas éticos de las trabajadoras y los trabajadores sociales en España". Papers. Revista de sociología, 97 (4): 875-898.

Banks, S. (2016): "Everyday ethics in professional life: social work as ethics work". Ethics and social welfare, 10 (1): 35-52.

Banks, S. y Williams, R. (2005): "Accounting for ethical difficulties in social welfare work: Issues, problems and dilemmas”. British journal of social work, (35): 1005-1022.

Bariffi, F. y Palacios, A. (2007): La discapacidad como una cuestión de derechos humanos. Una aproximación a la Convención Internacional sobre los Derechos de las Personas con Discapacidad. Madrid: Ediciones Cinca.

Beauchamp, T. (2016): "Chapter 1. Principlism in Bioethics”, en Serna, P. y Seoane, J. A. (eds.): Bioethical decision making and argumentation. Switzerland: Springer.

Beauchamp, T. (2010): Standing on principles. New York: Oxford University Press.

Beauchamp, T. y Childress, J. (2013): Principles of biomedical ethics. New York: Oxford University Press.

Bermejo, F. (2002): La ética del trabajo social. Bilbao: Desclée De Brouwer.

Bolívar, A. (2017): "Ética profesional en la formación y relaciones universitarias”, en Pérez, M. y Rodríguez, J. (coords.): Buenas prácticas docentes del profesorado universitario. Barcelona: Ediciones Octaedro.

Breakey, H. y Sampford, C. (2017): "Employed professionals' ethical responsibilities in public service and private enterprise: dilemma, priority and synthesis”. UNSW Law Journal, 40 (1): 262-300.

Brogna, P. (2009): "Las representaciones de la discapacidad: la vigencia del pasado en las estructuras sociales del presente", en Brogna, P. (comp.): Visiones y revisiones de la discapacidad. México: Fondo de Cultura Económica. 
Busso, C. y León-Sanz, P. (2016): "Investigación con pacientes en cuidados paliativos: dilemas éticos y percepción pública sobre su vulnerabilidad. Estudio exploratorio". Persona y Bioética, 20 (2): 132-150.

Cárcar, J. (2017): "La individualización institucionalizada y la asistencia sanitaria en relación a la Ley 41/2002, de 14 de noviembre, básica reguladora de la autonomía del paciente y de derechos y obligaciones en materia de información y documentación clínica su configuración". Bioderecho.es: Revista internacional de investigación en Bioderecho, (5): 1-20.

Carreón, J. (2015): "Compromiso laboral del trabajador social en el sector salud". Revista de contaduría y administración, 60 (1): 31-51.

Charlton, J. (1998): Nothing about us without us. Berkeley: University of California Press.

Cobo, J. (2001): Ética profesional en ciencias humanas y sociales. Madrid: Huerga y Fierro.

Comisión Económica para América Latina y el Caribe (CEPAL) (2017): Derechos de las personas mayores: retos para la interdependencia y autonomía. Santiago de Chile: CEPAL.

Comoretto, N. y Centeno-Cortés, C. (2016): "Experiences in clinical ethics. A project for meetings on clinical ethics in palliative medicine". Persona y bioética 20 (1): 38-47.

Congress, E. (2010): "Code of ethics", en Gray, M. y Webb, S. (eds.): Ethics and value perspectives in social work. London: Palgrave Macmillan.

Congress, E. y McAuliffe, E. (2006): "Social work ethics. Professional codes in Australia and the United States". International social work, 49 (2): 151-164.

Cubillos-Vega, C. (2017): "Análisis de la producción científica sobre Derechos Humanos en Trabajo Social: perspectiva internacional (2000-2015)". Revista Española de Documentación Científica, 40 (1): 1-10.

De la Cruz, G. (en prensa): "La inclusión de jóvenes con discapacidad auditiva en educación media superior: reconstruyendo la labor docente”, en Pérez-Castro, J. y López, A. (coords.): Discapacidad, inclusión social y educación.

Dhanda, A. (2008): "Construyendo un nuevo léxico de derechos humanos: La Convención sobre los Derechos de las Personas con Discapacidad”. Revista Internacional de derechos humanos, 5 (8): 42-59.

Díaz-Barriga, F. et al. (2016): "Para enseñar ética profesional no basta con una asignatura: los estudiantes de Psicología reportan incidentes críticos en aulas y escenarios reales". Revista iberoamericana de educación superior, 7 (18): 42-58.

Dolgoff, R. et al. (2012): Ehtical decisions for social work practice. Belmont: Brooks/Cole.

Encinas, A. (2014): "Aspectos teóricos de la ética profesional”. Lex: Revista de la Facultad de Derecho y Ciencia Política de la Universidad de Alas Peruanas, 12 (14): 319-336.

Etxeberría, X. (2016): "La docencia actual de la ética en las universidades jesuitas de España”. Arbor. Ciencia, pensamiento y cultura, 192 (782): 1-12.

Etxeberría, X. (2005): Aproximación ética a la discapacidad. Bilbao: Universidad de Deusto.

Etxeberría, X. et al. (2013): El enfoque ético del maltrato a las personas con discapacidad intelectual y del desarroIlo. Bilbao: Universidad de Deusto.

Félix, V. y Félix C. (2016): "Valores éticos en la formación del estudiante de psicología de la UAS". Revista iberoamericana de ciencias sociales y humanísticas, 5 (9): 1- 23. 
Fernández, T. y Ponce, L. (2014): “Naturaleza, filosofía, valores y principios del Trabajo Social con casos”, en Fernández, T. (coord.): Trabajo social con casos. Madrid: Alianza.

Ferrante, C. (2017): "El éxito de la Teletón en Chile: paradoja y mensaje en la era de los derechos". Acción Colectiva. Boletín Onteaiken, (23): 44-59.

Ferrante, C. (2015): “Discapacidad y mendicidad en la era de la Convención: ¿postal del pasado?”. Convergencia. Revista de ciencias sociales, 22 (68): 151-176.

Ferrante, C. y Ferreira, M. (2011): "Cuerpo y habitus: el marco estructural de la experiencia de la discapacidad". Intersticios: Revista sociológica de pensamiento crítico, 5 (2): 85-101.

Ferreira, M. (2007): "Prácticas sociales, identidad y estratificación: tres vértices de un hecho social, la discapacidad”. Intersticios. Revista sociológica de pensamiento crítico, 1 (2): 1-14.

Flores, C. y Martínez, G. (2006): "Hacia una concepción del Trabajo Social contemporáneo en México. Su condición profesional”. Revista Katálysis, 9 (2): 249-259.

French, S. y Swain, J. (2008): Understanding disability: A guide for health professionals. Churchill Livingstone: Elsevier.

Galeana, S. y Tello, N. (2010): "Fragilidad y debilidad del discurso en trabajo social: ausencia de la construcción desde la intervención social”. Revista Trabajo Social UNAM, (1): 22-35.

García, M. (2015): "El reconocimiento efectivo del derecho del menor a decidir sobre su salud". Bioderecho.es: Revista internacional de investigación en bioderecho, (2): 1-46.

Goffman, E. (1970): Estigma: la identidad deteriorada. Madrid: Amorrortu Editores.

González, E. (2017): "Valores en estudiantes de derecho. Proyección a la ética profesional: desarrollo de valores en universitarios". Revista internacional de ciencias humanas, 5 (1): 103-107.

Goodley, D. (2017): Disability Studies: An interdisciplinary introduction. London: Sage.

Grady, C. (2015): "Enduring and emerging challenges of informed consent". The new England Journal of Medicine, 372 (9): 855-862.

Grant, C. y Leigh, M. (2013): “The path of social justice: A human rights history of social justice education”. Equity \& excellence in education, 46 (1): 82-99.

Hernández, I. (2017): La importancia de la orientación a familias de adolescentes con discapacidad intelectual. Centro de Atención Múltiple Fray Pedro de Gante, Acajete, Puebla (tesis de licenciatura). Escuela de Licenciatura en Trabajo Social In-Vía: México.

Hirsch, A. y Navia, C. (2015): "Professional values training in the normal schools in Mexico in an uncertainty context", Edetania: estudios y propuestas socio-educativas, (47): 129-144.

Hirsch, A. y Pérez-Castro, J. (2013): "Estado de conocimiento sobre valores profesionales y ética profesional”, en Hirsch, A. y Yurén. T. (coords.): La investigación en México en el campo de Educación y Valores 2002 - 2011. México: ANUIES - COMIE.

Hortal, A. (2002): Ética general de las profesiones. Bilbao: Desclée de Brouwer.

Hortal, A. (1994): "Planteamiento de una ética profesional", en Fernández, J. L. y Hortal, A. (comps.): Ética de las profesiones. Madrid: Universidad Pontificia de Comillas. 
Idareta, F. et al. (2017): "150 años de historia de la ética del Trabajo Social en España: periodización de sus valores éticos". Cuadernos de trabajo social, 30 (1): 37-50.

Ife, J. (2012): Human rights and social work: Towards rights-based practice. Cambridge: Cambridge University Press.

Israel, M. (2015): Research ethics and integrity for social scientists. London: Sage.

Johnson, B. y Gore, N. (2016): "What do the professions 'profess'? Comparing architecture and planning codes of ethics". Architectural science review, 59 (6): 449-464.

Jorquera, C. (2014): "Ética profesional y derechos humanos: convergencias necesarias para la educación superior actual”. Temas de educación, 20 (2): 95-105.

Jurado, M. (2016): “La identidad profesional y ética, en la formación inicial de los estudiantes de la escuela normal de Naucalpan". Revista iberoamericana de producción académica y gestión educativa (5): 1-8.

Lázaro, E. y Silva, T. (2014): "El diagnóstico como estrategia para conocer la presencia de la discapacidad en la Escuela Nacional de Trabajo Social”. Revista Trabajo Social UNAM, (5): 77-92.

Lépiz, O. (2000): "El consentimiento informado y las personas con discapacidad mental”. Revista latinoamericana de derecho médico y medicina legal, 6 (1): 15-24.

Leturia, F. et al. (2014): "La atención sanitaria a las personas con discapacidad”. Revista Española de Discapacidad, 2 (1): 151-164.

Liamputtong, P. (2007): Researching the vulnerable: A guide to sensitive research methods. London: Sage.

Lidón, L. (2016): La discapacidad en el espejo y en el cristal: Derechos humanos, discapacidad y toma de conciencia, artículo 8 de la Convención Internacional sobre los Derechos de las Personas con Discapacidad, un camino previo por recorrer. Madrid: Ediciones CINCA.

López, A. (2016): “Ciudadanía, exclusión y vulnerabilidad en contextos trasnacionales”. Boletín de antropología. Universidad de Antioquía, 31 (52): 34-44.

López-Calva, J. (2015): "Socioética y antropoética profesional para una educación universitaria contra la desigualdad”. Edetania: estudios y propuestas socio-educativas, (47): 17-38.

López-Calva, J. (2014): "Ser persona y hacerse persona en el cambio de época: desafíos para la formación integral en la escuela y la universidad”. Revista latinoamericana de estudios educativos, 44 (3): 61-81.

López, J. et al. (2016): "Ética profesional y responsabilidad social en la Investigación: el caso de investigadores nacionales de una universidad privada en México", en Izarra, D. (coord.): Experiencias y perspectivas en ética profesional y responsabilidad social universitaria en Iberoamérica. Táchira, Venezuela: Universidad Pedagógica Experimental Libertador - Instituto de Mejoramiento Profesional del Magisterio.

López, P. et al. (2015): "El trabajo social y la inclusión educativa”. Margen: Revista de trabajo social y ciencias sociales, (79): 1-16.

Maggi, R. (1997): “Formación de valores y actitudes en la educación profesional técnica: caso CONALEP”. Revista latinoamericana de estudios educativos, 27 (1-2): 227-240.

Mancinas, S. et al. (2017): "Imaginarios e intervenciones de trabajadores sociales sobre familias con necesidades complejas en México y Suecia". Interacción y perspectiva, 7 (1): 37-60. 
Macheridis, N. y Paulsson, A. (2017): "Professionalism between profession and governance: how university teachers' professionalism shapes coordination". Studies in higher education, 0 (0): 1-16.

Mamo, L. y Fishman, J. (2013): "Why justice?: Introduction to the special issue of entanglements of science, ethics, and justice". Science, technology and human values, 38 (2): 159-175.

Molina, K. (2015): "Análisis de los derechos de las personas con discapacidad desde la teoría crítica". Anuario Centro de Investigación y Estudios Políticos, (6): 35-87.

Morris, J. (2001): "Impairment and disability: constructing and ethics of care that promotes human rights". Hypathia, 16 (4): 1-16.

Morris, J. (1991): Pride against prejudice. A personal politics of disability. London: Women’s Press Ltd.

Munuera, M. (2015): "The power of mediation's dialogue: resolution of conflicts in the environment of special needs people and/or people under functional dependence". Global journal of human-social science: C. Sociology \& culture, 15 (2): 13-22.

Munuera, M. (2012): "Trabajo social en la defensa de los derechos sociales de las personas con discapacidad". Trabajo social, (14): 93-104.

Munuera, M. y García-Longoria, M. (2013): "Ética del cuidado: empowerment y toma de decisiones”, en Rodón, L. y Taboada M. (coords.): Voces para la ética del trabajo social en tiempos trémulos. Madrid: Ediciones Paraninfo.

Muñoz, M. y Lucero, B. (2014): "Aspectos legales y bioéticos de intervenciones e investigaciones en personas con discapacidad intelectual en Chile". Acta Bioethica, 20 (1): 61-70.

Muñoz, C. y Rubio, M. (1993): Formación universitaria, ejercicio profesional y compromiso social. Resultados de un seguimiento de egresados de la Universidad Iberoamericana. México: UIA.

Naciones Unidas (2008): Convención sobre los Derechos de las Personas con Discapacidad. Serie de capacitación profesional no. 15. Nueva York: ONU.

Naciones Unidas (2006): Convención Internacional sobre los Derechos de las Personas con Discapacidad. Nueva York: ONU.

Navia, C. y Hirsch, A. (2015): "Ética profesional en estudiantes de posgrado en dos universidades mexicanas". Revista electrónica de investigación educativa, 17 (1): 100-115.

Palacios, A. (2017): "El modelo social de discapacidad y su concepción como cuestión de derechos humanos". Revista Colombiana de Ciencias Sociales, 8 (1): 14-18.

Palacios, A. (2008): El modelo social de discapacidad: orígenes, caracterización y plasmación en la Convención Internacional sobre los Derechos de las Personas con Discapacidad. Madrid: Ediciones Cinca.

Pantano, L. (2009): "Nuevas miradas en relación con la conceptualización de la discapacidad. Condición y situación de la discapacidad", en Brogna, P. (comp.): Visiones y revisiones de la discapacidad. México: Fondo de Cultura Económica.

Parrot, L. (2010): Values and Ethics in social work practice. London: Sage.

Pearson, H. et al. (2016): "The impact of Disability Studies curriculum on education professionals' perspectives and practice: Implications for education, social justice, and social change”. Disability studies quarterly, 36 (2).

Pérez, J. (2007): “Trabajo social: globalización y posmodernidad”. Revista internacional de ciencias sociales y humanidades, 17 (2): 151-173. 
Pérez-Castro, J. (2016): "La inclusión educativa desde la perspectiva de la responsabilidad social universitaria", en Arango, O. E. et al. (comps.): Ética profesional y responsabilidad social universitaria. Medellín: Fondo Editorial Luis Amigó.

Perlin, M. (2008): “'Everybody is making love/or else expecting rain’: considering the sexual autonomy. Rights of persons institutionalized because of mental disability in forensic hospitals and in Asia". Washington Law Review, 83 (481): 481-512.

Quinn, G. y Degener, T. (2002): Derechos humanos y discapacidad. Uso actual y posibilidades futuras de los instrumentos de derechos humanos de las Naciones Unidas en el contexto de la discapacidad. Nueva York: ONU.

Ramos, J. (2013): "Introducción a la perspectiva ética: principios prima facie, vulnerabilidad y competencia. Hacia una práctica de la salud mental basada en hechos y valores", en Plumed, C. (coord.): Ética y modelos de atención a las personas con transtorno mental grave. Madrid: Universidad Pontificia de Comillas - Fundación Juan Ciudad.

Reamer, F. (2014): “The Evolution of Social Work Ethics: Bearing Witness”, Advances in social work, 15 (1): 163-181.

Reamer, F. (2013): Social work values and ethics. Nueva York: Columbia University Press.

Resnik, D. (1998): The ethics of science. An introduction. Londres: Routledge.

Riddle, C. (2017): Human rights, disability, and capabilities. New York: Palgrave Macmillan.

Ríos, M. (1997): La socialización profesional de profesoras enfermeras de la UNAM (tesis de doctorado). Universidad Nacional Autónoma de México: México.

Rodríguez-Arias, D. y Rodríguez-Arias, J. (2005): “¿Deben decidir los «locos»?: Cuestiones sobre el consentimiento informado de los enfermos mentales en investigación clínica”, en Gómez-Heras, J. M. y Velayos, C. (edits.): Bioética. Perspectivas emergentes y nuevos problemas. Madrid: Tecnos.

Rodríguez, S. et al. (2017): "Cuerpo y emoción: la experiencia de la discapacidad en un mundo globalizado y neoliberal”, en De Sena, A. y Vergara, G. (comps.): Geometrías sociales. Buenos Aires: Estudios Sociológicos Editora.

Román, B. (2010): "La ética en los servicios de atención a las personas con discapacidad intelectual severa. ¿Por qué?”, en Fundación Víctor Grífols i Lucas (ed.): La ética en los servicios de atención a las personas con discapacidad intelectual severa. Barcelona: Fundación Víctor Grífols i Lucas.

Roncoli, V. (2017): "Vida cotidiana en discapacidad desde el trabajo social "Cómo se resignifica la vida cotidiana de la persona a partir de su condición/situación de discapacidad”. Revista Latinoamericana en discapacidad, sociedad y derechos humanos, 1 (1): 93-107.

Rueda, L. y Miranda, O. (2002): "Principales dilemas bioéticos en las personas con discapacidad prolongada". Acta bioética, 8 (1): 127-135.

Saks, M. (2016): "A review of theories of professions, organizations and society: The case for neo-Weberianism, neo-institutionalism and eclecticism". Journal of Professions and organization, 3 (2): 170-187.

Sandoval, H. et al. (2017): "Discapacidad en México: un análisis comparativo entre modelos descriptivos y periodos históricos mediante una línea del tiempo". Salud Publica de México, 59 (4): 429-436.

Schalock, R. (2015): "Las mejores prácticas de las organizaciones y los profesionales". Siglo cero: Revista española sobre Discapacidad intelectual, 46 (1): 7-23. 
Spitzer, T. (1990): El proceso de socialización del estudiante en la Universidad Autónoma de Chapingo. Hacia la internalización de un rol profesional (tesis de maestría). Cinvestav: México.

Tamayo, M. et al. (2017): "Determinantes sociales de la salud y discapacidad: actualizando el modelo de determinación”. Gac. sanit (s/n): 1-5.

Tapper, A. y Millett, S. (2014): “Is professional Ethics grounded in general ethical principles?”. Theoretical \& Applied Ethics, 3 (1): 61-80.

Tello, N. y Ornelas, A. (2015): Estrategias y modelos de intervención en trabajo social. México: Estudios de Opinión y Participación social A. C.

Uribe, E. y Romero, J. (2008): "Vulnerabilidad y victimización en el Estado mexicano". Espiral. Estudios sobre estado y sociedad, 14 (42): 75-95.

Úriz, M. J. et al. (2013): “¿Qué dilemas éticos encuentran los profesionales del trabajo social en España y cómo los resuelven?”. Revista de Treball Social. Collegi Oficial de Treball Social de Catalunya (199): 35-45.

Vanclay, F. et al. (2013): "Principles for ethical research involving humans: ethical professional practice impact assessments. Part I". Impact assessment and project appraisal, 31 (4): 243-253.

Venturiello, M. (2017): “Discapacitar”. Bordes. Revista de política, derecho y sociedad, 2 (5): 167-168.

Vera, M. et al. (2016): "Significado de los valores profesionales en docentes y estudiantes de enfermería, UNMSM - 2014". Anales de la Facultad de Medicina, 77 (3): 225-229.

Vila, E. y Cantero, F. (2017): "Ética profesional y derechos humanos: reflexiones y propuestas en el ámbito de la educación universitaria”, en Vila, E. (coord.): Competencias éticas y deontología profesional en la universidad. Málaga: Ediciones Aljibe.

Viscarret, J. et al. (2016): "Tipologías actuales de los trabajadores sociales en España". Cuadernos de trabajo social, 29 (2): 239-262.

Watson, N. et al. (eds.) (2012): Handbook of disability studies. Nueva York: Routledge.

Wilkinson, A. et al. (2016): "The changing world of professions and professional workers", en Wilkinson, A. et al. (eds.): Perspectives on contemporary professional work. Cheltenham, United Kingdom: Edward Elgar Publishing.

Yurén, T. et al. (2013): “Educación y valores. Formación del campo de investigación: avances y perspectivas”, en Hirsch, A. y Yurén. T. (coords.): La investigación en México en el campo de educación y valores 2002 - 2011. México: ANUIES - COMIE. 